msh-mss Mathématiques et sciences humaines

182 | Été 2008

Varia

\title{
Probabilités, propensions, utilités
}

Probabilities, propensities, utilities

\section{Stéphane Martin-Simig}

\section{OpenEdition}

Journals

Édition électronique

URL : http://journals.openedition.org/msh/10513

DOI : $10.4000 / \mathrm{msh} .10513$

ISSN : 1950-6821

\section{Éditeur}

Centre d'analyse et de mathématique sociales de l'EHESS

\section{Édition imprimée}

Date de publication : 30 juin 2008

Pagination : 17-31

ISSN : 0987-6936

\section{Référence électronique}

Stéphane Martin-Simig, «Probabilités, propensions, utilités », Mathématiques et sciences humaines [En ligne], 182 | Été 2008, mis en ligne le 30 juin 2008, consulté le 23 juillet 2020. URL : http:// journals.openedition.org/msh/10513; DOI : https://doi.org/10.4000/msh.10513 


\title{
PROBABILITÉS, PROPENSIONS, UTILITÉS
}

\author{
Stéphane MARTIN-SIMIG ${ }^{1}$
}

RÉSUMÉ - Nous montrons comment une théorie du consommateur, semblable à la théorie standard, peut être dérivée en remplaçant la notion d'utilité par une notion de propension, comprise comme la probabilité objective qu'un agent accomplisse un choix donné lorsqu'il est libre de contraintes. Nous montrons que cette propension peut se combiner avec l'existence d'une contrainte budgétaire et nous généralisons ensuite les résultats à la théorie du producteur.

MOTS CLÉS - Propension, Théorie du consommateur, Théorie des systèmes, Utilité.

SUMMARY - Probabilities, propensities, utilities

We show how a consumer theory, similar to the standard theory, can be derived by replacing the notion of utility with a notion of propensity, defined as an agent's objective probability of achieving a given choice in absence of constraints. We show that this propensity can be combined with budget contraints, and we generalize the results over the producer theory.

KEYWORDS - Consumer theory, Propensity, System theory, Utility.

La notion d'utilité comme principe d'action et de décision a connu bien des vicissitudes depuis sa formulation primitive au XVIII ${ }^{\mathrm{e}}$ siècle par Helvétius et les philosophes de l'illuminismo italien. Conçue à l'origine comme la mesure des sensations et des états psychiques agréables ou déplaisants d'un sujet ${ }^{2}$, elle a fini, de nos jours, par ne plus représenter souvent qu'une variable mathématique peu définie, un

\footnotetext{
${ }^{1}$ L'auteur est conseiller en analyse géopolitique et travaille principalement pour l'industrie privée, Les Douzilles 9, CH-1607 Les Thioleyres, Suisse, martin-simig@gmx.ch.

${ }^{2}$ La conception psychique-algédonique des proto-utilitaristes est par exemple apparente chez Helvétius dans des passages tels que : [...] si le desir du plaisir est le principe de toutes nos pensées et de toutes nos actions, si tous les hommes tendent continuellement vers leur bonheur réel ou apparent; toutes nos volontés ne sont donc que l'effet de cette tendance [Helvétius, 1758, discours 1, chap. 4] et la sensibilité physique est la cause unique de nos actions, de nos pensées, de nos passions, et de notre sociabilité. C'est pour se vêtir, pour parer sa maîtresse ou sa femme, leur procurer des amusements, nourrir soi et sa famille, et jouir enfin du plaisir attaché à la satisfaction des besoins physiques, que l'artisan et le paysan pensent, imaginent et travaillent. La sensibilité physique est donc l'unique moteur de l'homme [Helvétius, 1772, section 2, chap. 7], ainsi que chez Verri dans La sensibilità dell'uomo, il grande arcano, al quale è stata ridotta come a generale principio ogni azione della fisica sopra di noi, si divide, e scompone in due elementi, e sono amor del piacere, e fuga del dolore : tale almeno è la comune opinione degli uomini pensatori et maestri. Ognuno conosce e sente quanta influenza abbiano il piacere, e il dolore nel determinare le azioni umane; la speranza, il desiderio, il bisogno del primo; il timore, lo spavento, l'orrore del secondo danno il moto a tutte le nostre passioni [Verri, 1781, Introduzione].
} 
signe dans une formule. Censée résumer les préférences des agents, elle est invoquée fréquemment pour ne jouer que le rôle trop commode du quod maximandum qui permettra, selon l'observation de Samuelson ${ }^{3}$, de ramener (presque) tout problème économique à une affaire d'optimisation.

Dans la théorie standard du consommateur, l'utilité est essentiellement cette fonction qui, imputée à un sujet parfait devant allouer son budget entre des marchandises, permet de définir les quantités qu'il en acquerra à travers un calcul de maximisation. On suppose que le consommateur choisira son allocation de façon à obtenir la plus grande valeur possible de la fonction et, après quelques manipulations symboliques, on en tire la condition célèbre :

$$
\frac{U^{\prime}\left(Q_{x}\right)}{U^{\prime}\left(Q_{y}\right)}=\frac{p_{x}}{p_{y}}
$$

où $Q_{x}$ est la quantité achetée de la marchandise $x, Q_{y}$ celle de la marchandise $y, p_{x}$ et $p_{y}$ les prix des marchandises et $U^{\prime}\left(Q_{x}\right)$ et $U^{\prime}\left(Q_{y}\right)$ leurs utilités marginales pour le consommateur.

Le traitement de l'équilibre général n'est, pour ce qui nous concerne ici, guère différent : on impute aux ménages des fonctions d'utilité, qui ne sont souvent rien de plus que de simples lettres indicées, on admet que leur maximisation est soumise à certaines contraintes, et l'on se tire d'affaire par quelques opérations de calcul différentiel.

Ce n'est pas sans raison que l'utilité, dans son usage formel, est si peu définie. L'interprétation courante en fait une dimension subjective, une réalité qui n'est vraiment connaissable que par le seul sujet qui la ressent. Or, s'en servir pour élucider le choix du consommateur, c'est expliquer un phénomène aisément visible par le recours à des données inobservables. On comprend quel avantage il y aurait à remplacer la notion malheureusement intramentale d'utilité (ou celle de préférence ${ }^{4}$ ) par une autre qui serait plus directement expérimentale. L'échange contribuerait à dissiper les aspects antiempiriques de la théorie subjective de la valeur. Pour y parvenir, il faudrait d'emblée substituer à l'explication des comportements comme résultats de phénomènes psychiques internes aux agents - et ainsi cachés dans les

\footnotetext{
${ }^{3}$ Pour une discussion du principe du maximum de Samuelson et de son importance dans la formalisation et la résolution des problèmes de l'économie politique ordinaire, on se reportera à [Samuelson, 1970].

${ }^{4}$ Nous évoquons ici surtout la conception de l'utilité comme une fonction, mais ce que nous en disons s'applique aussi bien à l'utilité définie sous la forme de préférences. Substituer des ordres de préférences aux fonctions d'utilité (ordinales) ne change guère l'affaire au fond. Un ordre de préférences n'est qu'une autre façon de représenter une famille de courbes d'utilité homothétiques, comme une fonction d'utilité n'est qu'une recette pour développer des préférences ordonnées. Qui connaît l'une déduit les autres, et qui attribue des préférences aux agents leur attribue aussi - sans y songer peut-être - les fonctions qui les résument.

Si les préférences sont immédiatement observables, leur émergence ne peut s'expliquer qu'en supposant dans les agents un principe générateur, une faculté de préférer dont elles tirent leur source. Or ce principe joue un rôle formellement équivalent à celui de l'utilité dans sa conception moderne, et n'est pas moins mystérieux. On s'abstient le plus souvent d'y faire allusion, mais on n'obtient alors qu'une théorie incomplète, qui borne son domaine aux données empiriques parce qu'elle n'attaque qu'une partie de son problème.
} 
replis inaccessibles de la conscience - une explication construite sur des propriétés objectivables, comme des probabilités.

Nous appellerons théorie propensive l'approche consistant à dériver les lois des comportements de cette hypothèse que les agents sont doués de propensions, c'està-dire de probabilités objectives (et a priori, si les circonstances s'y prêtent, déductibles d'observations) d'accomplir certains actes, sans que ces probabilités soient expliquées par référence à des sentiments, ou à des systèmes internes de préférence, ni à quelle que sorte que ce soit d'entités intrasubjectives. Nous voulons expliquer les décisions et les choix des sujets humains de la même façon que l'on explique les événements stochastiques qui affectent les corps inanimés. Les probabilités dont nous parlons sont des attributs physiques de l'automate humain, au même titre que les probabilités de mouvement des molécules d'un gaz, par exemple, ou que, dans un autre domaine, les probabilités d'apparition des numéros de roulette.

Si nous parvenons à déduire de la notion de propension les déterminations des prix et des quantités, nous pourrons dire que nous aurons réduit l'utilité, comprise alors au sens large de moteur des actions économiques, à cette autre notion plus objective, ou, ce qui revient en fait au même, que nous aurons donné le moyen de substituer la seconde à la première.

\section{LA THÉORIE PROPENSIVE DU CONSOMMATEUR}

Nous supposons qu'au cours d'une période constituée d'une succession de $T$ instants, un consommateur dispose d'un budget de $R$ unités monétaires. Il s'en sert pour acheter deux marchandises $x$ et $y$, vendues aux prix $p_{x}$ et $p_{y}$. Nous admettons qu'il dépense la totalité de $R$ au cours de $T$. Ce consommateur est doué d'une propension $P r_{x}$ à acheter du $x$ et $P r_{y}$ à acheter du $y$. Par propension, nous entendons la probabilité (au sens mathématique) d'acheter une unité de $x$ ou une unité de $y$ au cours d'un instant quelconque en l'absence de contraintes et de perturbations adventices. Nous supposons que les instants sont suffisamment brefs pour qu'il ne soit possible d'acheter qu'une seule unité de marchandise (ou aucune) au cours de chacun.

Si nous faisons abstraction pour un moment de la contrainte de budget, nous pouvons poser que la probabilité que le consommateur achète un nombre $K_{x}$ d'unités de $x$ est donnée par une loi de Poisson de moyenne $P r_{x} T$. Nous avons affaire en effet à un événement (l'achat de $x$ ) dont nous savons qu'il se produira en moyenne $\operatorname{Pr}_{x} T$ fois au cours d'une période $T$, et nous nous enquérons de la probabilité qu'il se produise $K_{x}$ fois au cours d'une période donnée. En admettant que $K_{x}$ soit assez grand, nous pouvons approcher cette probabilité par une loi normale $N\left(m_{x} ; s_{x}\right)$ de moyenne $m_{x}=\operatorname{Pr}_{x} T$ et d'écart type $s_{x}=\sqrt{\operatorname{Pr}_{x} T}$.

Du fait des propriétés usuelles de la loi normale, cette probabilité est également expressible comme $\left(1 / s_{x}\right) \cdot \varphi\left(\left(K_{x}-m_{x}\right) / s_{x}\right)$, où $\varphi(a)$ est la valeur prise en $a$ par la loi normale centrée réduite. La probabilité que le consommateur acquière $K_{x}$ unités 
de $x$ est donc de $\left(1 / \sqrt{\operatorname{Pr}_{x} T}\right) \cdot \varphi\left(\left(K_{x}-\operatorname{Pr}_{x} T\right) / \sqrt{\operatorname{Pr}_{x} T}\right)$ en l'absence de contraintes ${ }^{5}$.

Pour les mêmes raisons, la probabilité que le consommateur achète $K_{y}$ unités du bien $y$ en l'absence de contraintes pourra être approchée par une loi normale $\left(1 / s_{y}\right) \cdot \varphi\left(\left(K_{y}-m_{y}\right) / s_{y}\right)=\left(1 / \sqrt{P r_{y} T}\right) \cdot \varphi\left(\left(K_{y}-P r_{y} T\right) / \sqrt{P r_{y} T}\right)$. La probabilité qu'il achète, au cours de la période, un panier constitué de $K_{x}$ unités de $x$ et de $K_{y}$ unités de $y$ sera donnée par le produit de ces deux probabilités simples : $\left(1 / s_{x}\right)$. $\varphi\left(\left(K_{x}-m_{x}\right) / s_{x}\right) \cdot\left(1 / s_{y}\right) \cdot \varphi\left(\left(K_{y}-m_{y}\right) / s_{y}\right)$.

Or le consommateur n'est pas seulement mû par ses propensions à acheter, il est aussi contraint par les limites de son budget et les prix des biens. Nous nous intéressons ici au comportement normal et ordinaire d'un consommateur quelconque. Nous souhaitons connaître son choix le plus probable. La théorie propensive du consommateur cherchera à déterminer la loi d'allocation qui décrit la répartition du budget entre les achats avec la plus grande probabilité.

Celle-ci peut se trouver aisément en recherchant quel panier $\left\{K_{x} ; K_{y}\right\}$ a la plus grande probabilité d'être choisi, compte cette fois tenu de la contrainte budgétaire. Nous voulons déterminer

$$
\operatorname{Max} \frac{1}{s_{x}} \cdot \varphi\left(\frac{K_{x}-m_{x}}{s_{x}}\right) \cdot \frac{1}{s_{y}} \cdot \varphi\left(\frac{K_{y}-m_{y}}{s_{y}}\right) \text {, sous la contrainte } R=p_{x} K_{x}+p_{y} K_{y}
$$

Ceci ne présente pas de problèmes particuliers : le lagrangien est

$$
L=\left(\frac{1}{s_{x}} \cdot \varphi\left(\frac{K_{x}-m_{x}}{s_{x}}\right) \cdot \frac{1}{s_{y}} \cdot \varphi\left(\frac{K_{y}-m_{y}}{s_{y}}\right)\right)-\lambda\left(R-p_{x} K_{x}-p_{y} K_{y}\right)
$$

En posant sa dérivée par $K_{x}$ égale à zéro, nous obtenons

$$
\frac{d \varphi\left(\frac{K_{x}-m_{x}}{s_{x}}\right)}{d K_{x}} \cdot \frac{1}{s_{x}} \cdot \frac{1}{s_{y}} \cdot \varphi\left(\frac{K_{y}-m_{y}}{s_{y}}\right)=\frac{d \lambda\left(R-p_{x} K_{x}-p_{y} K_{y}\right)}{d K_{x}}
$$

Cependant,

$$
\frac{d \varphi\left(\frac{K_{x}-m_{x}}{s_{x}}\right)}{d K_{x}}=\frac{d \varphi(u)}{d u} \cdot \frac{d u}{d K_{x}}
$$

\footnotetext{
${ }^{5}$ Nous approximons une fonction de masse par une fonction de densité. Ceci peut sembler problématique au premier abord, mais ce qui compte ici en fait, c'est que $\left(1 / s_{x}\right) \varphi\left(\left(K_{x}-m_{x}\right) / s_{x}\right)$ fournit, au moins pour certaines valeurs entières de $K_{x}$, une approximation passable de $\left(\left(P r_{x} T\right)^{K_{x}} e^{-P r_{x} T}\right) /\left(K_{x}\right.$ !), et qu'elle offre l'avantage de la continuité, permettant qu'on lui applique des techniques simples d'optimisation. Le procédé se justifie aussi par la simplification qu'il apporte au calcul : comme nous le verrons dans la suite, la loi normale possède certaines propriétés qui se révèlent utiles lorsqu'il s'agit de la dériver.

Si l'on préfère, on peut aussi poser la probabilité d'achat comme $P\left(K_{x} \leq X_{K_{x}} \leq K_{x}+d K_{x}\right) \approx$ $\left(\left(1 / \sqrt{P r_{x} T}\right) \cdot \varphi\left(\left(K_{x}-\operatorname{Pr}_{x} T\right) / \sqrt{\operatorname{Pr}_{x} T}\right)\right) d K_{x}$. Si la variation minimale $d K_{x}$ est égale à une unité, il en vient $P\left(K_{x} \leq X_{K_{x}} \leq K_{x}+1\right) \approx\left(1 / \sqrt{P r_{x} T}\right) \cdot \varphi\left(\left(K_{x}-P r_{x} T\right) / \sqrt{P r_{x} T}\right)$; et même si $d K_{x} \neq 1$, elle doit disparaître au cours des calculs qui suivent, n'ayant pas, pour ce qui nous importe ici, d'impact sur les résultats.
} 
avec $u=\left(\left(K_{x}-m_{x}\right) / s_{x}\right)$.

Or c'est une propriété de la loi normale centrée réduite $\varphi(a)$ que sa dérivée en $a$ est $-a \varphi(a)$; donc

$$
\frac{d \varphi(u)}{d u}=-u \varphi(u)=-\frac{K_{x}-m_{x}}{s_{x}} \cdot \varphi\left(\frac{K_{x}-m_{x}}{s_{x}}\right)
$$

En outre,

$$
\frac{d u}{d K_{x}}=\frac{d\left(\frac{K_{x}-m_{x}}{s_{x}}\right)}{d K_{x}}=\frac{1}{s_{x}}
$$

Donc

$$
\frac{d \varphi\left(\frac{K_{x}-m_{x}}{s_{x}}\right)}{d K_{x}}=-\frac{K_{x}-m_{x}}{s_{x}} \cdot \varphi\left(\frac{K_{x}-m_{x}}{s_{x}}\right) \cdot \frac{1}{s_{x}}
$$

La condition de maximisation pour $x$ devient finalement

$$
\frac{K_{x}-m_{x}}{s_{x}^{3}} \cdot \varphi\left(\frac{K_{x}-m_{x}}{s_{x}}\right) \cdot \frac{1}{s_{y}} \cdot \varphi\left(\frac{K_{y}-m_{y}}{s_{y}}\right)=\lambda p_{x}
$$

Pour que nous ayons affaire à un maximum, la dérivée seconde du lagrangien doit en outre être négative :

$$
\frac{d\left(\left(-\frac{K_{x}-m_{x}}{s_{x}^{2}} \cdot \frac{1}{s_{x}} \cdot \varphi\left(\frac{K_{x}-m_{x}}{s_{x}}\right) \cdot \frac{1}{s_{y}} \cdot \varphi\left(\frac{K_{y}-m_{y}}{s_{y}}\right)\right)+\lambda p_{x}\right)}{d K_{x}}<0
$$

Ce qui revient à

$$
\begin{aligned}
& \left(\frac{d\left(\frac{K_{x}-m_{x}}{s_{x}^{2}}\right)}{d K_{x}} \cdot \frac{1}{s_{x}} \cdot \varphi\left(\frac{K_{x}-m_{x}}{s_{x}}\right) \cdot \frac{1}{s_{y}} \cdot \varphi\left(\frac{K_{y}-m_{y}}{s_{y}}\right)\right)+ \\
& \left(\frac{K_{x}-m_{x}}{s_{x}^{2}} \cdot \frac{1}{s_{x}} \cdot \frac{d \varphi\left(\frac{K_{x}-m_{x}}{s_{x}}\right)}{d K_{x}} \cdot \frac{1}{s_{y}} \cdot \varphi\left(\frac{K_{y}-m_{y}}{s_{y}}\right)\right)>0
\end{aligned}
$$


Comme nous savons déjà que

$$
\frac{d \varphi\left(\frac{K_{x}-m_{x}}{s_{x}}\right)}{d K_{x}}=-\frac{K_{x}-m_{x}}{s_{x}^{2}} \cdot \varphi\left(\frac{K_{x}-m_{x}}{s_{x}}\right)
$$

et comme

$$
\frac{d\left(\frac{K_{x}-m_{x}}{s_{x}^{2}}\right)}{d K_{x}}=\frac{1}{s_{x}^{2}}
$$

nous aurons

$$
\begin{aligned}
& \left(\frac{1}{s_{x}^{2}} \cdot \frac{1}{s_{x}} \cdot \varphi\left(\frac{K_{x}-m_{x}}{s_{x}}\right) \cdot \frac{1}{s_{y}} \cdot \varphi\left(\frac{K_{y}-m_{y}}{s_{y}}\right)\right)- \\
& \quad\left(\frac{K_{x}-m_{x}}{s_{x}^{2}} \cdot \frac{K_{x}-m_{x}}{s_{x}^{2}} \cdot \frac{1}{s_{x}} \cdot \varphi\left(\frac{K_{x}-m_{x}}{s_{x}}\right) \cdot \frac{1}{s_{y}} \cdot \varphi\left(\frac{K_{y}-m_{y}}{s_{y}}\right)\right)>0
\end{aligned}
$$

qui se simplifie en

$$
s_{x}^{2}>\left(K_{x}-m_{x}\right)^{2}
$$

Comme, par ailleurs, $s_{x}^{2}=m_{x}=P r_{x} T$, la condition du second ordre sera

$$
\operatorname{Pr}_{x} T>\left(K_{x}-\operatorname{Pr}_{x} T\right)^{2}
$$

Si nous faisons le même raisonnement dans le cas de la marchandise $y$, nous devons obtenir les conditions analogues

$$
\frac{K_{y}-m_{y}}{s_{y}^{3}} \cdot \varphi\left(\frac{K_{y}-m_{y}}{s_{y}}\right) \cdot \frac{1}{s_{x}} \cdot \varphi\left(\frac{K_{x}-m_{x}}{s_{x}}\right)=\lambda p_{y}
$$

et

$$
\operatorname{Pr}_{y} T>\left(K_{y}-\operatorname{Pr}_{y} T\right)^{2}
$$

avec $m_{y}=s_{y}^{2}=\operatorname{Pr}_{y} T$.

En divisant les deux conditions de premier ordre pour $x$ et pour $y$ l'une par l'autre, nous avons

$$
\frac{\frac{K_{x}-m_{x}}{s_{x}^{3}} \cdot \varphi\left(\frac{K_{x}-m_{x}}{s_{x}}\right) \cdot \frac{1}{s_{y}} \cdot \varphi\left(\frac{K_{y}-m_{y}}{s_{y}}\right)}{\frac{K_{y}-m_{y}}{s_{y}^{3}} \cdot \varphi\left(\frac{K_{y}-m_{y}}{s_{y}}\right) \cdot \frac{1}{s_{x}} \cdot \varphi\left(\frac{K_{x}-m_{x}}{s_{x}}\right)}=\frac{p_{x}}{p_{y}}
$$


$\mathrm{Ou}$

$$
\frac{K_{x}-P r_{x} T}{K_{y}-P r_{y} T} \cdot \frac{P r_{y} T}{P r_{x} T}=\frac{p_{x}}{p_{y}}
$$

Finalement, les conditions qui définissent la loi d'allocation du budget du consommateur au cours d'une période $T^{6}$ dans le cas où le choix est limité à deux biens seront

1. $R=p_{x} K_{x}+p_{y} K_{y}$

2. $\frac{\frac{K_{x}}{P r_{x}}-T}{\frac{K_{y}}{P r_{y}}-T}=\frac{p_{x}}{p_{y}}$

3. $\operatorname{Pr}_{x} T>\left(K_{x}-\operatorname{Pr}_{x} T\right)^{2}$

4. $\operatorname{Pr}_{y} T>\left(K_{y}-\operatorname{Pr}_{y} T\right)^{2}$

Ces conditions se généralisent bien entendu aisément au cas d'un consommateur devant allouer son budget entre un nombre quelconque de marchandises : Avec $n$ marchandises $K_{1}, K_{2}, \ldots, K_{n}$ offertes à l'achat et un budget de $R$, le lagrangien du problème s'écrira

$$
\begin{aligned}
L= & \frac{1}{s_{1}} \cdot \varphi\left(\frac{K_{1}-m_{1}}{s_{1}}\right) \cdot \frac{1}{s_{2}} \cdot \varphi\left(\frac{K_{2}-m_{2}}{s_{2}}\right) \cdot \ldots \cdot \\
& \frac{1}{s_{n}} \cdot \varphi\left(\frac{K_{n}-m_{n}}{s_{n}}\right)-\lambda\left(R-p_{1} K_{1}-p_{2} K_{2}-\ldots-p_{n} K_{n}\right)
\end{aligned}
$$

L'annulation de sa dérivée par $K_{i}$ et $K_{j}$ quelconques donnera

$$
\frac{1}{s_{1}} \cdot \varphi\left(\frac{K_{1}-m_{1}}{s_{1}}\right) \cdot \frac{1}{s_{2}} \cdot \varphi\left(\frac{K_{2}-m_{2}}{s_{2}}\right) \cdot \ldots \cdot \frac{1}{s_{n}} \cdot \varphi\left(\frac{K_{n}-m_{n}}{s_{n}}\right) \cdot \frac{K_{i}-m_{i}}{s_{i}^{2}}=\lambda p_{i}
$$

et

$$
\frac{1}{s_{1}} \cdot \varphi\left(\frac{K_{1}-m_{1}}{s_{1}}\right) \cdot \frac{1}{s_{2}} \cdot \varphi\left(\frac{K_{2}-m_{2}}{s_{2}}\right) \cdot \ldots \cdot \frac{1}{s_{n}} \cdot \varphi\left(\frac{K_{n}-m_{n}}{s_{n}}\right) \cdot \frac{K_{j}-m_{j}}{s_{j}^{2}}=\lambda p_{j}
$$

En divisant ces deux expressions l'une par l'autre nous aurons donc

$$
\frac{K_{i}-P r_{i} T}{K_{j}-P r_{j} T} \cdot \frac{P r_{j}}{P r_{i}}=\frac{p_{i}}{p_{j}}
$$

Au bout du compte, dans le cas où $n$ marchandises sont offertes à l'achat et où le consommateur dispose d'un budget de $R$, la contrainte de budget, le ratio des prix et les conditions de second ordre deviennent

\footnotetext{
${ }^{6}$ On peut éliminer $T$ de cette formule si l'on choisit les unités de telle sorte que $T=1$. Cette remarque vaut aussi naturellement pour les autres formules analogues.
} 
1. $R=p_{1} K_{1}+p_{2} K_{2}+\ldots+p_{n} K_{n}$

2. $\frac{\frac{K_{i}}{P r_{i}}-T}{\frac{K_{j}}{P r_{j}}-T}=\frac{p_{i}}{p_{j}}$, pour toute paire de marchandises $i$ et $j$,

3. $\operatorname{Pr}_{i} T>\left(K_{i}-\operatorname{Pr}_{i} T\right)^{2}$, pour toute marchandise $i$.

La seconde expression ne met en jeu, outre les propensions, que les grandeurs observables des prix et des quantités. Il est donc aisé, connaissant les secondes, de déduire les premières. Deux observations distinctes du ratio des prix et du couple des quantités de deux marchandises consommées par un agent suffiront pour calculer ses propensions, au prix de l'hypothèse de leur permanence à moyen terme. Le système d'équations

$$
\begin{aligned}
& \frac{K_{i}}{P r_{i}}-T=\frac{p_{i}}{p_{j}} \cdot\left(\frac{K_{j}}{P r_{j}}-T\right) \\
& \frac{K_{i}^{\prime}}{P r_{i}}-T^{\prime}=\frac{p_{i}^{\prime}}{p_{j}^{\prime}} \cdot\left(\frac{K_{j}^{\prime}}{P r_{j}}-T^{\prime}\right)
\end{aligned}
$$

ne possède en effet qu'une seule solution pour $\left\{P r_{i} ; P r_{j}\right\}^{7}$.

Comme au reste les propensions ne sont vraiment que des probabilités objectives, elles se laisseront déterminer empiriquement à l'instar des probabilités de n'importe quels évènements. Il ne sera pas plus difficile, au moins quant à la théorie, d'identifier à peu près la probabilité qu'un consommateur débourse tant d'unités de numéraire au cours d'une période dans l'acquisition de telle marchandise que de connaître par l'expérience avec quelle chance une pièce donnée retombe sur pile.

La relation entre le ratio des prix et les quantités consommées se prête à la comparaison avec la relation standard $U^{\prime}\left(K_{i}\right) / U^{\prime}\left(K_{j}\right)=p_{i} / p_{j}$ dès qu'est stipulée la forme prise par $U$ dans cette dernière. $\mathrm{Si}$, par exemple et comme on fait souvent, nous voulons lui donner celle d'une fonction de Cobb-Douglas, $U\left(K_{i} ; K_{j}\right)=K_{i}^{\alpha} \cdot K_{j}^{\beta} \cdot \gamma$, nous aurons

$$
\frac{\alpha}{\beta} \cdot \frac{K_{j}}{K_{i}}=\frac{p_{i}}{p_{j}}
$$

La quantité consommée de $i$ en fonction des autres grandeurs (notamment le ratio des prix) sera

$$
K_{i}=\frac{\alpha}{\beta} \cdot \frac{K_{j}}{p_{i} / p_{j}}
$$

dans le modèle standard et

\footnotetext{
${ }^{7}$ les deux valeurs successives de $K_{i}, K_{j}, p_{i}, p_{j}$ et $T$ sont connues, $P r_{i}$ et $P r_{j}$ sont les inconnues.
} 


$$
K_{i}=P r_{i} \cdot \frac{p_{i}}{p_{j}} \cdot \frac{K_{j}}{P r_{j}}-P r_{i} T \cdot \frac{p_{i}}{p_{j}}+P r_{i} T
$$

dans le modèle propensif. Les deux modèles prédisent comme on voit des structures de consommation fort différentes entre lesquelles les données empiriques feront le partage. Une utilité à la Cobb-Douglas fait de $K_{i}$ une grandeur proportionnelle à $K_{j}$ et inversement proportionnelle au ratio des prix ; le jeu des propensions donne à la relation des trois variables - tout autre facteur demeurant constant — une forme caractéristique et distincte. Il en ira de même de la comparaison avec une fonction d'utilité quelconque, sauf à choisir celle-ci telle qu'elle singe justement la structure propensive, supposition trop visiblement ad hoc pour qu'on la prenne au sérieux.

La connaissance des propensions offre le moyen de calculer la probabilité d'un choix de consommation, la probabilité du choix d'un panier donné étant le produit des probabilités de ses ingrédients. Ce sera du moins le cas des paniers composés de biens dont les propensions sont indépendantes ${ }^{8}$.

Ces probabilités sont des grandeurs cardinales et deux conséquences remarquables en découlent : d'abord, qu'il est possible de se livrer à des comparaisons intersubjectives et de déterminer dans quelle mesure tel consommateur est plus favorisé qu'un autre dans l'expression de ses propensions par l'ordre économique en vigueur. Ensuite, qu'on peut estimer l'écart entre le choix constaté et le choix idéal de l'agent, c'est-à-dire le choix qu'il réaliserait libéré d'une contrainte de budget ${ }^{9}$.

Le choix idéal est donné par l'optimisation du lagrangien

$$
L=\frac{1}{s_{1}} \cdot \varphi\left(\frac{K_{1}-m_{1}}{s_{1}}\right) \cdot \frac{1}{s_{2}} \cdot \varphi\left(\frac{K_{2}-m_{2}}{s_{2}}\right) \cdot \ldots \cdot \frac{1}{s_{n}} \cdot \varphi\left(\frac{K_{n}-m_{n}}{s_{n}}\right)
$$

La condition de sa maximisation sera

soit

$$
\frac{K_{i}}{P r_{i}}-T=\frac{K_{j}}{P r_{j}}-T
$$

$$
\frac{K_{i}}{K_{j}}=\frac{P r_{i}}{P r_{j}}
$$

\footnotetext{
${ }^{8}$ On ne peut exclure a priori que des propensions soient mutuellement dépendantes, que la propension d'un agent à choisir un bien soit une probabilité conditionnelle de son choix d'un autre bien. Certains des $P r_{i}$ mentionnés dans le lagrangien de l'agent seraient alors des fonctions des $K_{j}$, ce qui compliquerait quelque peu sa dérivation. Seule l'expérience pourra décider de la pertinence de cette hypothèse. Nous nous en tenons ici à l'indépendance des propensions parce qu'elle constitue le cas le plus simple, qui mérite d'être exploré d'abord, et parce qu'en l'absence de raisons suggérant le contraire, c'est elle qu'on devra préférer.

${ }^{9} \mathrm{Ou}$ si les prix des marchandises étaient nuls et leur abondance infinie, ce qui revient au même. Déterminer les conditions de l'optimum en faisant fictivement abstraction de toute contrainte fournit ainsi un critère de l'optimum optimorum pour un consommateur. Nous verrons plus loin que ce critère s'étend sans peine à un groupe de sujets.
} 
qui peut ainsi, lorsqu'elle est considérée en même temps qu'un ratio des quantités empiriques, servir de référence pour juger de la distorsion créée dans la structure de consommation d'un agent par la contrainte de budget et l'existence des prix. Plus le ratio effectif $K_{i} / K_{j}$ s'éloigne de $\mathrm{Pr}_{i} / P r_{j}$, plus la distorsion est forte et plus les contraintes économiques entravent le sujet dans la manifestation de ses propensions.

\section{LA MACROCONSOMMATION PROPENSIVE}

Le raisonnement précédent, développé en ne considérant qu'un seul consommateur, peut aussi bien s'étendre au cas des choix de consommation de groupes d'agents ou d'une économie entière. Une analyse conduite dans le cadre des propensions échappe à la dichotomie du macro et du micro-niveau. Peut-être du reste le concept de propension consomptive paraîtra-t-il ainsi encore plus naturel que dans le cas du consommateur individuel.

Si nous supposons données les propensions collectives à consommer de telle ou telle marchandise, nous pouvons nous en servir pour déterminer non seulement les allocations budgétaires (c'est-à-dire les choix de quantité), mais les prix eux-mêmes.

Supposons, pour simplifier le modèle, qu'une économie ne connaisse qu'une seule ressource, présente en quantité $R$ et apte à produire une pluralité de $n$ marchandises dans les quantités respectives $K_{1}, K_{2}, \ldots, K_{n}$ selon des fonctions de production $f_{1}\left(R_{1}\right)=K_{1}, f_{2}\left(R_{2}\right)=K_{2}, \ldots, f_{n}\left(R_{n}\right)=K_{n}$. En admettant que le budget agrégé des consommateurs soit également $R$, que ceux-ci soient animés collectivement par les propensions $\mathrm{Pr}_{1}, \mathrm{Pr}_{2}, \ldots, \mathrm{Pr}_{n}$ (où $\mathrm{Pr}_{i}$ est la probabilité que l'un ou l'autre des consommateurs achète une unité de $i$ au cours d'un des (très) brefs instants constituant la période), et que les firmes obéissent à l'impératif équimarginaliste d'égalité des prix et des coûts à la marge et vendent leurs marchandises aux prix $p_{1}$, $p_{2}, \ldots, p_{n}$, nous aurons :

1. Pour toute paire de firmes productrices des marchandises $k$ et $l^{10}$ :

$$
\frac{f_{k}^{\prime}\left(R_{k}\right)}{f_{l}^{\prime}\left(R_{l}\right)}=\frac{p_{l}}{p_{k}}
$$

2. Pour toute paire de marchandises $i$ et $j$ :

$$
\frac{\frac{K_{i}}{P r_{i}}-T}{\frac{K_{j}}{P r_{j}}-T}=\frac{p_{i}}{p_{j}}
$$

\footnotetext{
${ }^{10} \mathrm{La}$ firme productrice de la marchandise $k$ doit maximiser son profit $p_{k} f_{k}\left(R_{k}\right)-R_{k} p_{r}$ (où $p_{r}$ est le prix de la ressource). Le programme de cette maximisation est Max $p_{k} f_{k}\left(R_{k}\right)-R_{k} p_{r}$. La condition de l'optimum s'obtient en annulant la dérivée du profit par $R_{k}: p_{k} f_{k}^{\prime}\left(R_{k}\right)-R_{k}^{\prime} p_{r}=0$, soit $p_{k} f_{k}^{\prime}\left(R_{k}\right)=p_{r}$. En appliquant le même raisonnement à la production de la marchandise $l$, on obtient $p_{l} f_{l}^{\prime}\left(R_{l}\right)=p_{r}$. D'où $p_{k} f_{k}^{\prime}\left(R_{k}\right)=p_{l} f_{l}^{\prime}\left(R_{l}\right)$, et $f_{k}^{\prime}\left(R_{k}\right) / f_{l}^{\prime}\left(R_{l}\right)=p_{l} / p_{k}$.
} 
3. Pour toute marchandise $i$ :

$$
\operatorname{Pr}_{i} T>\left(K_{i}-\operatorname{Pr}_{i} T\right)^{2}
$$

4. $K_{1} p_{1}+K_{2} p_{2}+\ldots+K_{n} p_{n}=R_{1}+R_{2}+\ldots+R_{n}=R$

Les commentaires développés plus haut sur les choix propensifs du consommateur individuel se transposent au consommateur collectif, nous n'avons guère besoin d'y insister. La fixité des propensions sur un terme assez étendu semble encore plus recevable lorsqu'elle s'applique à des groupes d'agents; elles expriment alors des attributs institutionnels qui font le compromis des propensions de tous les participants.

La détermination des propensions collectives peut aussi servir à construire un référentiel normatif pour confronter la structure de consommation de l'économie à la structure idéale qui se réaliserait si la société pouvait donner libre cours à ses tendances, sans des contraintes à respecter. Nous avons vu que la condition de l'optimum sans contrainte est $K_{i} / K_{j}=P r_{i} / P r_{j}$. Interprétée au macro-niveau, elle indique les proportions dans lesquelles la production des marchandises devrait s'effectuer idéalement. Jointe aux conditions de l'usage optimal des facteurs, elle offre la règle pour légiférer sur les choix allocatifs et montre dans quel sens les politiques publiques devront s'appliquer à rectifier une structure de consommation effective pour l'améliorer.

\section{LES PROPENSIONS DES PRODUCTEURS}

Nous avons supposé dans le paragraphe précédent que les producteurs étaient (classiquement) animés par un objectif de maximisation des profits, au contraire des consommateurs, dont nous faisions les jouets de leurs propensions. Cette double hypothèse n'est pas nécessairement contradictoire.

S'il est peu convaincant d'imputer à des consommateurs de chair et d'os un comportement trop calculateur et omniscient, il ne serait pas absurde d'imaginer que des firmes, créations artificielles, en soient plus rapprochées. Elles pourraient être organisées de façon à réussir dans des tâches auxquelles l'esprit des simples humains n'est pas approprié.

Cependant, puisque nous avons voulu dans ces pages montrer comment le comportement des consommateurs peut s'expliquer à travers l'hypothèse des propensions objectives, il paraît naturel de s'en servir pour élucider aussi celui des producteurs. Dans un tel cas, les décisions d'achat de facteurs de la part des producteurs (c'està-dire l'allocation de leurs recettes) dépendront, elles aussi, de leurs propensions.

Si nous imaginons une économie dans laquelle une pluralité de $c$ consommateurs allouent leurs budgets entre $n$ biens de consommation offerts par $n$ producteurs employant $m$ facteurs, et que ces producteurs sont, comme les consommateurs, animés de propensions de forme $\operatorname{Pr}_{u v}$ (où $u$ représente le sujet de la propension (consommateur ou producteur) et $v$ la marchandise à l'égard de laquelle elle s'exerce), cette économie vérifiera les relations suivantes : 
1. Pour toute paire de biens de consommation $i$ et $j$ et tout consommateur $x$ :

$$
\frac{\frac{K_{x i}}{P r_{x i}}-T}{\frac{K_{x j}}{P r_{x j}}-T}=\frac{p_{i}}{p_{j}}
$$

où $K_{x i}$ et $K_{x j}$ sont les quantités de $i$ et de $j$ achetées par $x$.

2. Pour toute paire de facteurs $k$ et $l$ et tout producteur $i^{11}$ :

$$
\frac{\frac{F_{i k}}{P r_{i k}}-T}{\frac{F_{i l}}{P r_{i l}}-T}=\frac{q_{k}}{q_{l}}
$$

où $F_{i k}$ et $F_{i l}$ représentent les quantités de facteurs employées par $i$ et $q_{k}$ et $q_{l}$ leurs prix.

3. Pour tout consommateur $x$ :

$$
R_{x}=p_{1} K_{x 1}+p_{2} K_{x 2}+\ldots+p_{n} K_{x n}
$$

où $R_{x}$ est le budget du consommateur $x$.

4. Pour tout producteur $i$ :

$$
p_{i} f_{i}\left(F_{i 1} ; F_{i 2} ; \ldots ; F_{i m}\right)=q_{1} F_{i 1}+q_{2} F_{i 2}+\ldots+q_{m} F_{i m}
$$

où $p_{i}$ est le prix du produit $i$ mis sur le marché par le $i^{\mathrm{e}}$ producteur et $f_{i}(\ldots)$ sa fonction de production donnant le volume du produit qu'il réalise avec ses facteurs.

5. Pour tout produit $i$ :

$$
f_{i}\left(F_{i 1} ; F_{i 2} ; \ldots ; F_{i m}\right)=K_{1 i}+K_{2 i}+\ldots+K_{c i}
$$

où $f_{i}\left(F_{i 1} ; F_{i 2} ; \ldots ; F_{i m}\right)$ est la quantité totale de $i$ mise sur le marché par son fabricant, et où les $K_{v i}$ sont les quantités de $i$ achetées par les $c$ consommateurs.

6. Pour tout facteur $k$ :

$$
F_{k}=F_{1 k}+F_{2 k}+\ldots+F_{r k}
$$

où $F_{u k}$ est la quantité de $k$ acquise et employée par le $u^{\mathrm{e}}$ producteur et $F_{k}$ une constante $^{12}$.

\footnotetext{
${ }^{11}$ Nous donnons indifféremment les mêmes indices aux variables relatives à un produit et à celles qui se rapportent à son producteur.

${ }^{12}$ Dans ce paragraphe, nous supposons simplement donnés les budgets des consommateurs et les quantités de facteurs, sans nous interroger sur leur provenance. La distribution des revenus est toujours dépendante des arrangements institutionnels en vigueur, et ceux-ci varient selon les sociétés. Les revenus peuvent provenir d'un droit sur les facteurs ou de transferts étatiques, ou d'autres sources. Nous préférons laisser cette question ouverte puisqu'elle n'a pas directement de portée sur notre propos; libre au lecteur d'ajouter des relations pour préciser ce point, s'il le souhaite.
} 
7. Pour tout consommateur $x$ et tout produit $i$ :

$$
\operatorname{Pr}_{x i} T>\left(K_{x i}-\operatorname{Pr}_{x i} T\right)^{2}
$$

8. Pour tout producteur $i$ et tout facteur $k$ :

$$
\operatorname{Pr}_{i k} T>\left(K_{i k}-\operatorname{Pr}_{i k} T\right)^{2}
$$

L'usage fait de leurs facteurs par des producteurs propensifs diverge de celui qu'ils manifesteraient s'ils étaient mus par un impératif de maximisation de leur profit, comme on le leur impute dans le modèle standard. Cette différence, analogue à celle que nous avons constatée dans le comportement des consommateurs, implique que le rendement marginal d'un facteur ne sera pas normalement égalisé avec son prix. Hors une coïncidence invraisemblable, les quantités de deux facteurs injectées dans un procès de production ne respecteront pas la condition $f_{i}^{\prime}\left(F_{i k}\right) / f_{i}^{\prime}\left(F_{i l}\right)=q_{k} / q_{l}$, et les producteurs n'assureront pas une allocation optimale au sens où d'ordinaire l'on entend l'optimalité ${ }^{13}$.

Un modèle du producteur voulant pour ses prémisses que les agents maximisent leurs propensions sous une contrainte s'écarte ainsi notablement du modèle standard dans ses prédictions, comme le modèle propensif du consommateur s'écarte du modèle utilitarien.

L'hypothèse des propensions rend compte de la conduite des agents sans référence à leur rationalité, et sans nous contraindre à trancher s'ils s'appuient dans leurs choix sur une raison absolue, limitée, biaisée ou située. Elle suppose seulement qu'ils sont dotés de probabilités de devenir les sujets de tel évènement ou de tel autre, comme l'est toute entité du monde physique. N'exigeant pas d'autres axiomes que ceux des probabilités, déjà donnés dans la mathématique, et n'introduisant pas de nouveau principe, elle se montre plus parcimonieuse et générale que l'analyse standard, qui doit poser l'échafaudage de ses postulats spécifiques et définir ses propres concepts avant de dérouler le fil de son argument.

D’une théorie des préférences révélées, elle se distingue par son univocité et sa plus grande précision. La théorie des préférences révélées peut montrer quel ensemble de courbes d'indifférence sied à des vecteurs de prix et de quantités connus empiriquement, mais elle ne va pas jusqu'à en spécifier une seule. Dans le cas ordinaire, c'en est une infinité, rationalisables en autant de fonctions d'utilité, qui resteront compatibles avec un nombre fini d'observations. Il faut une longue série de données pour connaître assez bien un ordre de préférence, quand deux suffisent pour identifier les propensions sur une paire de marchandises.

Trouver dans la maximisation de probabilités sous des contraintes les lois du mouvement économique rend possible de dériver analytiquement leurs propriétés pertinentes et de les fonder. La théorie standard se contente d'imposer aux fonctions d'utilité — ou aux ordres de préférences — certaines conditions formelles ${ }^{14}$, mais elle

\footnotetext{
${ }^{13}$ La théorie propensive offre l'avantage subsidiaire de prémunir la notion d'équilibre économique contre la tentation des interprétations déontiques. L'équilibre que définit l'optimisation sous contrainte des propensions est bien, dans le sens mathématique du mot, un optimum, mais il ne court guère le danger qu'on le prenne aussi pour un optimum moral.

${ }^{14}$ Comme d'être dérivables ou concaves.
} 
ne produit guère les motifs nécessaires et décisifs pour lesquels celles-là devraient les posséder ${ }^{15}$. Qu'on leur attribue souvent la forme de fonctions de Cobb-Douglas, qui parmi d'autres satisfont ces conditions, n'écarte l'obstacle qu'au prix d'un arbitraire de plus. Les produits de lois statistiques suivent en revanche une forme définie ${ }^{16}$, fixée par leur nature. Et les propriétés qu'on peut leur découvrir ont leurs racines dans une raison plus ferme que la commodité technique.

Ceci débouche sur une théorie de la valeur objective, puisque fondée sur les propensions des agents, à l'opposé du subjectivisme des théories de la valeur tirées des utilités marginales ou bâties sur la supputation d'un ordre de préférences présent dans l'esprit de chaque sujet.

On comprend que les agents puissent parfois choisir à rebours de leurs intérêts ou de leur plaisir, puisque leurs propensions n'y sont pas nécessairement conformes. Cette dissociation couramment constatée des conduites et des intérêts constitue un autre écart avec la doctrine standard, et apporte à l'interprétation propensive une confirmation supplémentaire, quand même elle ne se plie pas à la rigueur d'une quantification.

\section{LES SYSTÈMES PROPENSIFS}

La théorie propensive du consommateur n'est qu'un cas particulier de la théorie des systèmes propensifs (comme, du reste, celle du producteur). Si par système propensif à contraintes on entend un ensemble de variables $X_{1}, X_{2}, \ldots, X_{n}$ telles que la probabilité que $X_{i}$ prenne la valeur a est approximativement donnée par $p r_{i}(a)\left(P\left(X_{i}=a\right) \approx p r_{i}(a)\right)^{17}$, mais qui sont justement sujettes aux $m$ contraintes $f_{1}\left(x_{1} ; x_{2} ; \ldots ; x_{n}\right) \leq 0, f_{2}\left(x_{1} ; x_{2} ; \ldots ; x_{n}\right) \leq 0, \ldots, f_{m}\left(x_{1} ; x_{2} ; \ldots ; x_{n}\right) \leq 0$, on pourra supposer que l'état normal (le plus probable ou à peu près) d'une variable $X_{i}$ dans un tel système sera donné par l'application des conditions classiques de Kuhn-Tucker ${ }^{18}$ :

1. $f_{h}\left(x_{1} ; x_{2} ; \ldots ; x_{n}\right) \leq 0$, pour tous les $h$,

2. $\lambda_{h} f_{h}\left(x_{1} ; x_{2} ; \ldots ; x_{n}\right)=0$, pour tous les $h$,

\footnotetext{
${ }^{15}$ Tout au plus invoque-t-on la nécessité de donner à la doctrine un tour apte au traitement mathématique, et qui garantisse l'existence d'une solution et son unicité. Mais c'est faire des conséquences qu'on en tire ensuite des pétitions de principe. On ne saisit guère pourquoi des réalités psychiques comme les préférences devraient se plier à ces conditions, sinon que la démonstration l'exige. Qu'en revanche les probabilités d'acquisition de quantités au cours d'une période dessinent une loi de Poisson (ou approximativement une gaussienne) procède de leur nature de probabilités.

${ }^{16}$ Cette forme est définie même si l'on choisit de remplacer les courbes exactes par des approximations.

${ }^{17}$ Nous nous préoccupons principalement ici, comme du reste dans les pages qui précèdent, des cas où $X_{i}$ ne peut prendre que des valeurs discrètes, mais il est également possible de considérer les cas dans lesquels elle serait continue. $p r_{i}(a)$ doit alors être définie comme la fonction donnant la probabilité que $X_{i}$ prenne une valeur comprise dans un certain intervalle autour de $a: p r_{i}(a) \approx$ $P\left(a-\varepsilon \leq X_{i} \leq a+\varepsilon\right)$. Sous cette réserve, les énoncés qui suivent demeurent pertinents.

${ }^{18}$ Nous devons naturellement supposer que les fonction composant le lagrangien $L=\left(p r_{1}\left(x_{1}\right)\right.$. $\left.p r_{2}\left(x_{2}\right) \cdot \ldots \cdot p r_{n}\left(x_{n}\right)\right)-\lambda_{1} f_{1}\left(x_{1} ; x_{2} ; \ldots ; x_{n}\right)-\lambda_{2} f_{2}\left(x_{1} ; x_{2} ; \ldots ; x_{n}\right)-\ldots-\lambda_{m} f_{m}\left(x_{1} ; x_{2} ; \ldots ; x_{n}\right)$ sont telles que celui-ci est dérivable et qu'en outre la fonction objectif $p r_{1}\left(x_{1}\right) \cdot p r_{2}\left(x_{2}\right) \cdot \ldots \cdot p r_{n}\left(x_{n}\right)$ est quasi-concave cependant que les contraintes sont convexes.
} 
3. $\lambda_{h} \geq 0$, pour tous les $h$,

4. $\frac{d\left(p r_{1}\left(x_{1}\right) \cdot p r_{2}\left(x_{2}\right) \cdot \ldots \cdot p r_{n}\left(x_{n}\right)\right)}{d x_{i}}=\lambda_{1} \frac{d f_{1}\left(x_{i}\right)}{d x_{i}}+\lambda_{2} \frac{d f_{2}\left(x_{i}\right)}{d x_{i}}+\ldots+\lambda_{m} \frac{f_{m}\left(x_{i}\right)}{d x_{i}}$.

L'état normal du système dans son entier sera décrit par la généralisation de ces conditions à l'ensemble des variables.

Puisque les systèmes propensifs à contraintes peuvent décrire aussi bien le comportement de systèmes d'êtres animés que d'êtres inanimés, la théorie du consommateur (et, avec elle, la théorie de bien des faits socioéconomiques) doit être tenue pour un cas parmi d'autres dans une famille de problèmes probabilistes. L'analyse propensive est susceptible d'applications au-delà du domaine de l'économique pure, elle peut se transposer mutatis mutandis à tout ensemble d'évènements probables soumis à des contraintes.

Remerciements. L'auteur exprime sa gratitude aux deux évaluateurs anonymes qui ont fourni leurs commentaires d'une première version de cet article.

\section{BIBLIOGRAPHIE}

BARBerÀ S., HAMmond P.J., SEIDl C., Handbook of utility theory, Vol. I et II, Dordrecht, Kluwer cop., 1998.

Helvétius C.A., De l'esprit, 1758, [consultable en ligne : http ://gallica.bnf.fr/].

Helvétius C.A., De l'homme, de ses facultés intellectuelles, et de son éducation, 1772, [consultable en ligne : http ://gallica.bnf.fr/].

SAMUelson P.A., Maximum principles in analytical economics, Nobel Memorial Lecture, 1970 .

VERri P., Discorsi del conte Pietro Verri sull'indole del piacere e del dolore, sulla felicità e sulla economia politica, 1781, [consultable en ligne : http ://gallica.bnf.fr/]. 